\title{
X-ray absorption-based imaging and its limitations in the differentiation of ancient mummified tissue
}

\author{
Journal Article \\ Author(s): \\ Wanek, Johann; Székely, Gábor; Rühli, Frank \\ Publication date: \\ 2010-05 \\ Permanent link: \\ https://doi.org/10.3929/ethz-b-000030137
}

Rights / license:

In Copyright - Non-Commercial Use Permitted

Originally published in:

Skeletal Radiology 40(5), https://doi.org/10.1007/s00256-010-1035-9 


\title{
X-ray absorption-based imaging and its limitations in the differentiation of ancient mummified tissue
}

\author{
Johann Wanek • Gábor Székely • Frank Rühli
}

Received: 11 May 2010/Revised: 27 August 2010/Accepted: 8 September 2010/Published online: 12 January 2011

(C) ISS 2010

\begin{abstract}
Objectives Differentiation of ancient tissues is of key importance in the study of paleopathology and in the evolution of human diseases. Currently, the number of imaging facilities for the non-destructive discrimination of dehydrated tissue is limited, and little is known about the role that emerging imaging technologies may play in this field. Therefore, this study investigated the feasibility and quality of dual-energy computed tomography (DECT) for the discrimination of dry and brittle soft tissue. Moreover, this study explored the relationship between morphological changes and image contrast in ancient tissue by using X-ray micro-tomography (micro-CT).

Materials and Methods An Egyptian mummy head and neck was scanned with DECT at tube voltage/current of $140 \mathrm{kVp} / 27 \mathrm{mAs}$ (tube A) and $100 \mathrm{kVp} / 120 \mathrm{mAs}$ (tube B). The CT attenuation was determined by regions of interest (ROI) measurements of hard and soft tissue of the mummy skull. Finally, two samples from the posterior neck were dissected to acquire micro-CT images of shrunken dehydrated tissue.
\end{abstract}

J. Wanek · F. Rühli $(\bowtie)$

Centre for Evolutionary Medicine, Institute of Anatomy,

University of Zurich,

Winterthurerstrasse 190,

8057 Zurich, Switzerland

e-mail: frank.ruhli@anatom.uzh.ch

J. Wanek

e-mail: johann.wanek@anatom.uzh.ch

G. Székely

Computer Vision Laboratory, ETH Zentrum,

Sternwartstrasse 7,

8092 Zurich, Switzerland

e-mail: szekely@vision.ee.ethz.ch
Results Dual-energy CT images demonstrated the high contrast resolution of surface structures from mummy skull. Bone density changes in the posterior skull base as well as soft-tissue alterations of the eyes and tongue were assessed. Micro-CT scans allowed the identification of morphological changes and the discrimination of muscle tissue from inorganic material in samples taken from the neck.

Conclusions Significant attenuation differences $(p<0.0007)$ were observed within 12 of the 15 ancient tissue groups and organic materials using DECT. We detected a correlation between X-ray scattering and image contrast reduction in dehydrated tissue with micro-CT imaging.

Keywords Dual-energy CT $\cdot$ Micro computed tomography Tissue discrimination

\section{Introduction}

Mummification has a long history in many cultures around the world, with preservation considered to be essential for the survival of the deceased in the afterlife [1]. Despite the advancements in imaging methods, the diagnostic imaging of mummified remains is still a logistical and technological challenge for the paleopathologist. The material properties of the dehydrated tissue have changed significantly in comparison to normal tissue, particularly with regard to density and homogeneity. Taking this into account, the radiological differentiation and interpretation of compact and morphologically changed tissue layers requires highly skilled researchers. In the past, $\mathrm{CT}$ has been successfully used for the non-invasive imaging of mummified corpses [2]. Computed tomography (CT) of mummies began in 1977 
[3]; in that report Lewin and Harwood Nash investigated the internal structure of mummified tissue, because some human diseases can be discovered in preserved ancient soft tissues rather than from ancient skeletal tissue [4].

Due to the fact that conventional single-source CT suffers from low soft tissue differentiation in paleoradiology, there is an increased interest in novel imaging techniques such as dual-energy computed tomography (DECT). The use of DECT imaging may foster the identification of paleopathological features or alterations.

In clinical practice, material discrimination using DECT is successfully applied for the differentiation of organic and inorganic materials, e.g. urinary stones [5]. Dual-source CT is routinely used in the clinical environment for abdominal imaging [6] and has been successfully introduced into the CT angiography of abdominal aortic aneurysms. In general, DECT offers more specific tissue characterisation than CT and could improve the assessment of diseases for paleopathological investigations [7].

Since the early 1980s, micro-CT has become a powerful research method that is ideally suited to complementing the existing capabilities of histology as discussed in [8]. In paleoradiology, micro-CT imaging is extremely helpful in studying the morphological alterations of ancient tissue and in assessing quality aspects of X-ray absorption-based imaging.

The aim of this study was to assess the spatial tissue discrimination in ancient remains using the Hounsfield values of DECT imaging. Micro-CT imaging was performed to explore the correlation between morphological changes and image contrast of mummified tissue.

\section{Materials and methods}

Subject description and sample preparation

An isolated head and neck of an Egyptian mummy (dated approximately 1000 B.C. from the former collection of the Musée d'Orbe in Switzerland) was used for this study. The subject (Fig. 1) is wrapped in 12 layers of thin gauze-like linen. For detailed tomographic examination using the X-ray micro-CT we dissected two samples (sample 1, sample 2) to investigate the deep neck muscles, see the marked region in Fig.1a.

\section{Dual-energy CT imaging}

Dual-energy CT studies of the mummy head were performed at the University Hospital in Zurich. Scans from the wrapped mummified head were made using dual-source $\mathrm{CT}$ in dual-energy mode (SOMATOM Definition Dual Source, CT SOM5 SPI DUAL; Siemens, Germany). The

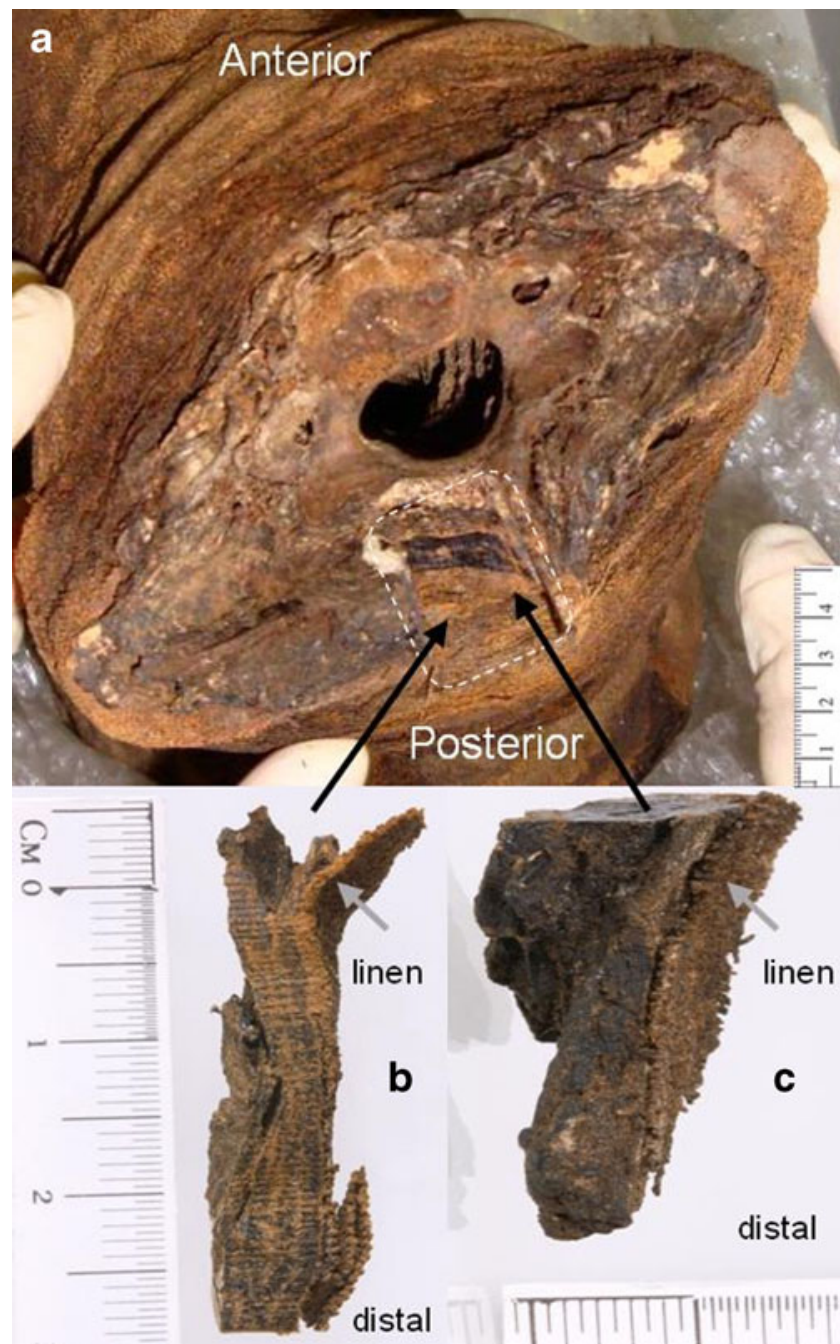

Fig. 1 a Photograph of an Egyptian mummy head showing a cross section of the neck at the level of C6. The area of dissection is indicated as dotted rectangle. $\mathbf{b}$, c Photographs of the dissected neck sample 1 (b) and sample 2 (c) illustrate the original linen layers in the distal direction

head was laid in the supine position and the energy of the two X-ray tube voltages was varied independently within the range $80 \mathrm{kVp}$ and $140 \mathrm{kVp}$. All images were collected in a sequential scanning mode (using a single source). Higher energies were used to reduce noise in the images. Images were reconstructed with an overlapping slice thickness of $0.6 \mathrm{~mm}$ (increment $0.4 \mathrm{~mm}$ ) and a matrix size of $512 \times 512$.

The pixel spacing of the detector was $0.42 \mathrm{~mm}$. The HU values of the mummy head were assessed on a PC workstation (Intel ${ }^{\circledR}$ Core ${ }^{\mathrm{TM}} 2$ Quad Processor $2.4 \mathrm{GHz}$, 8 GB RAM, NVIDIA ${ }^{\circledR}$ Quadro FX3800, Microsoft Windows 7 Professional: 64-bit edition) using freely available software from Image Information Systems Ltd, Germany (IQ-View PRO V1.0.8 V1.1.04.) 


\section{Micro-CT imaging}

Two tissue samples (samples 1 and 2) from the neck region were taken to acquire images by using a highresolution desktop cone-beam scanner (SkyScan 1174; SkyScan, Kontich, Belgium). Micro-CT images were acquired at the Paul Scherrer Institute in Villigen, Switzerland. The device was equipped with an X-ray CCD camera, a cooled 1,024 $\times 1,024$-pixel 12-bit detector with a nominal spatial resolution of $27 \mu \mathrm{m}$. The size of the samples was about $6 \mathrm{~mm}$ in diameter with a length of about $20 \mathrm{~mm}$. All samples were mounted on a turntable stage that rotates the specimen in small increments up to a total rotation of $180^{\circ}$.

The volumetric reconstruction of the raw data was performed using Nrecon software (SkyScan). The reconstruction algorithm allows corrections of beam-hardening and ring artefacts. The data sets for each sample were analysed using SkyScan CT analyser software.

\section{Tissue classification}

In order to classify the findings, we divided the tissue discrimination into three main groups according to tissue density and the size of samples, such as cortical bone, trabecular bone and embalming material (group 1), tongue and philtrum representing soft tissue layers with small attenuation differences (group 2) and micro-anatomical tissue discrimination of neck muscles such as sample 1 and sample 2 (group 3).

A comparative analysis was made between the groups (1 and 2) using DECT imaging and the Mann-Whitney significance test. Group 3 was evaluated using micro-CT imaging. Density measurements in Hounsfield units [HU] from DECT imaging were taken from regions of interest (ROI), for instance, the tongue, philtrum, trabecular bone, cortical bone and embalming substance.

\section{Statistical analysis}

The non-parametric Kruskal-Wallis test was performed to compare the mean $\mathrm{CT}$ attenuation differences between the groups tongue, linen, philtrum, embalming material, trabecular bone and cortical bone, which were measured at $80 \mathrm{kVp}$ and $140 \mathrm{kVp}$. The post-hoc Mann-Whitney test was required after we completed the Kruskal-Wallis test in order to determine which groups differ from each other. For the statistical significance test, we used the differences in Hounsfield units (i.e. $\Delta \mathrm{HU}=\mathrm{HU}_{80 \mathrm{kV}}-\mathrm{HU}_{140 \mathrm{kV}}$ ); such as tongue $\left(\Delta \mathrm{HU}_{\mathrm{T}}\right)$, linen $\left(\Delta \mathrm{HU}_{\mathrm{L}}\right)$, philtrum $\left(\Delta \mathrm{HU}_{\mathrm{P}}\right)$, embalming material $\left(\Delta \mathrm{HU}_{\mathrm{EM}}\right)$, trabecular bone $\left(\Delta \mathrm{HU}_{\mathrm{TB}}\right)$ and cortical bone $\left(\Delta \mathrm{HU}_{\mathrm{CB}}\right)$.

The $p$ values were corrected using the Bonferroni method for 15 group comparisons (Table 1) according to the binomial equation $\{\mathrm{n} ! /(\mathrm{k} !(\mathrm{n}-\mathrm{k}) !)\}=15$, with $n=6$ for six tissue types and $\mathrm{k}=2$.

According to Bonferroni, a significance level $p<0.01 / 15$ or $p<0.0007$ was considered statistically significant. Data are presented as mean \pm standard deviation (SD) or median \pm percentile. The statistical analysis was conducted using $\mathrm{R}$ software for Linux ( $\mathrm{R}$ version 2.9.2; the $\mathrm{R}$ Foundation for Statistical Computing).

\section{Results}

Hard tissue differentiation by DECT using a single energy

By using single-energy scans, rough structures with large HU differences such as cortical, trabecular and parietal bone can be well distinguished.

The parietal bone in Fig. 2 showed a higher X-ray attenuation with a mean HU value of 630 (minimum 391, maximum 1026) around the region of embalming material and lambdoidal suture in comparison to the region of the sagittal suture with a mean $\mathrm{HU}$ value of $-197 \mathrm{HU}$ (minimum -412 , maximum 305).

Hard and soft tissue differentiation

Figure 3 depicts the coronal CT showing an excellent state of preservation of the eyes and tongue. Also apparent is the loss of volume of these organs due to the dehydration process by mummification.

Table 1 Tissue group comparisons

\begin{tabular}{|c|c|c|c|c|}
\hline $\begin{array}{l}\text { Tongue } \\
\text { compared with }\end{array}$ & $\begin{array}{l}\text { Linen } \\
\text { compared with }\end{array}$ & $\begin{array}{l}\text { Philtrum } \\
\text { compared with }\end{array}$ & $\begin{array}{l}\text { Embalming material } \\
\text { compared with }\end{array}$ & $\begin{array}{l}\text { Trabecular bone } \\
\text { compared with }\end{array}$ \\
\hline Linen philtrum & Philtrum & Embalming material & Trabecular bone & Cortical bone \\
\hline Embalming material & Embalming material & Trabecular bone & Cortical bone & \\
\hline Trabecular bone & Trabecular bone & Cortical bone & & \\
\hline Cortical bone & Cortical bone & & & \\
\hline
\end{tabular}




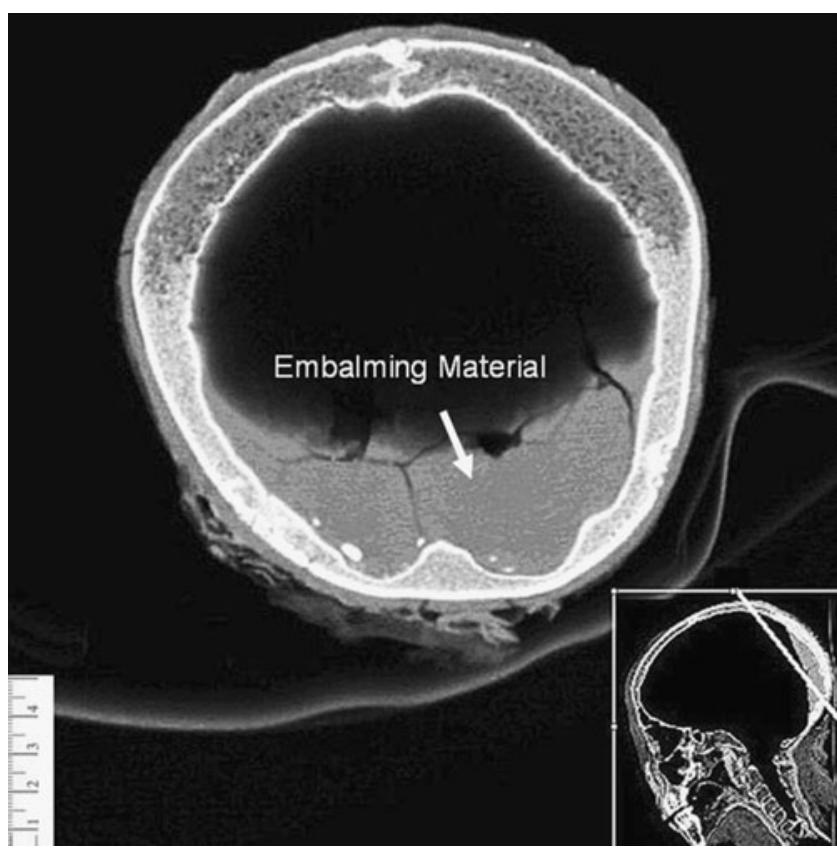

Fig. 2 Volume-rendered computed tomography image of the mummy skull depicts the potential of hard tissue discrimination. The brain was removed post-mortem. Note the deposits of higher-Z embalming substance in the posterior skull base. The insert shows the section plane of the main image. Tube voltage $140 \mathrm{kVp}$, tube current $27 \mathrm{mAs}$

The volumetric image in Fig. 3 qualitatively demonstrates the hard and soft tissue differentiation by using a single energy. Rough structures can be well distinguished from the background.

Tissue differentiation using Hounsfield units and dual energy

Two different energies of DECT allow a clear differentiation of tissues, which possess small differences in $\mathrm{HU}$ values with a single energy, such as the tongue, the philtrum and the embalming material (compare Table 2, $80 / 140 \mathrm{kVp}$ ). We found an overlap of $\mathrm{HU}$ values in the measurements for the embalming material, tongue, philtrum, similar to that depicted in Motley et al. [9].

The attenuation values at different tube voltages, for a minimum of 20 ROIs, are summarized in Table 2. The wrapped linen was not strongly attenuating the X-rays, which is demonstrated by the low HU values in Table 2 [10]. The smaller tube voltage of $80 \mathrm{kVp}$ led to an increase in the attenuation and larger HU differences.

Figure 4 illustrates the mean $\mathrm{HU}$ values of mummified remains for the groups such as the linen, philtrum, tongue and embalming material. The mean CT interval between the groups was about $100 \mathrm{HU}$ and was calculated by using the formula: $\Delta \mathrm{HU}_{\text {mean }}=\mathrm{HU}_{80 \mathrm{kV}}-\mathrm{HU}_{140 \mathrm{kV}}$.

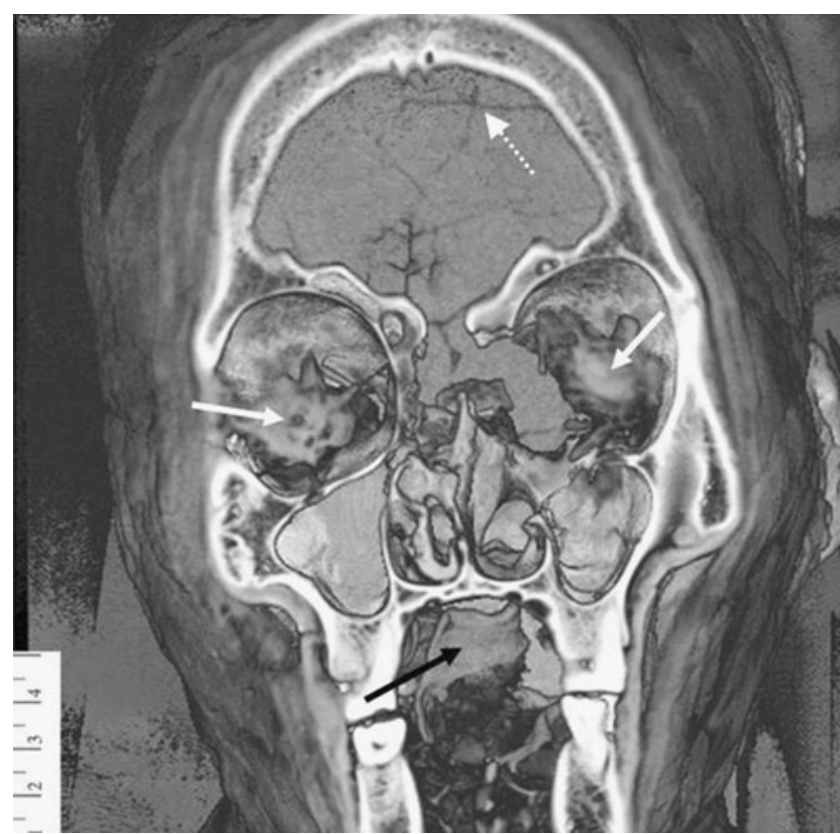

Fig. 3 Volume-rendered coronal view of the mummy skull obtained by CT. The image shows soft tissue remains of the eyes (solid white arrows) and tongue (black arrow). Note the cracks of resin in the posterior skull base (dotted white arrow). Tube voltage $140 \mathrm{kVp}$, tube current $27 \mathrm{mAs}$

In 12 out of 15 cases, the attenuation differences among the six groups L, P, TB, EM, T and CB were, according to the 15 paired post-hoc tests, statistically significant $(p<0.0007)$. No significant difference $(p>0.0007)$ was noted between the CT values for $\{\mathrm{T}, \mathrm{CB}\}\{\mathrm{T}, \mathrm{TB}\}$ and $\{\mathrm{EM}, \mathrm{TB}\}$, because of the higher measurement uncertainties of the tissue groups $\mathrm{CB}$ and TB. Four tissue groups with significant attenuation differences were depicted in Fig. 5.

As expected, hard and soft tissue differentiation (group 1) of the mummy head was excellent for the imaging of ancient tissue by DECT. Soft tissue discrimination between the tongue and philtrum (group 2) was good for both imaging methods. However, the tissue discrimination was limited by overlapping and ambiguous HU values (Fig. 5). Clearly, the tissue

Table 2 Mean CT attenuation values at $80 \mathrm{kVp}$ and $140 \mathrm{kVp}$ of various tissue types in the mummy head

\begin{tabular}{lll}
\hline Tissue type & Attenuation \\
\cline { 2 - 3 } & $\begin{array}{ll}80 \mathrm{kVp} \\
\mathrm{HU}(\text { mean } \pm \mathrm{SD})\end{array}$ & $\begin{array}{l}140 \mathrm{kVp} \\
\mathrm{HU}(\text { mean } \pm \mathrm{SD})\end{array}$ \\
\hline Linen & $222 \pm 52$ & $-296 \pm 73$ \\
Tongue & $493 \pm 65$ & $108 \pm 36$ \\
Philtrum & $179 \pm 41$ & $90 \pm 27$ \\
Embalming material & $380 \pm 48$ & $121 \pm 28$ \\
\hline
\end{tabular}

SD: standard deviation with $n=20$; HU: Hounsfield units 




Fig. 4 The diagram demonstrates the tissue-specific attenuation scanned with dual-energy CT at $80 \mathrm{kVp}$ and $140 \mathrm{kVp}$ tube voltage. Data points reflect the mean and standard deviation (vertical and horizontal lines) of four different measurements. The capital letters represent $\mathrm{HU}$ values measured on the mummy head, i.e. $L$ linen, $P$ philtrum, $T$ tongue, $E M$ embalming material

discrimination of microanatomy (group 3) is not feasible with DECT imaging.

\section{Micro-CT assessment of ancient tissue}

The mummification process leads to various physical alterations within the tissues, which were studied using micro-CT. As shown in Fig. 6, the texture analysis of the specific tissue architecture could not be performed. The

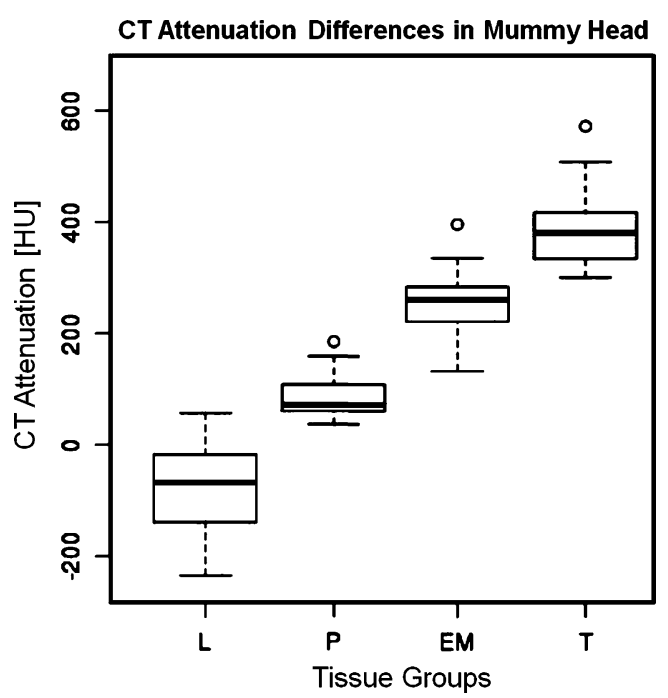

Fig. 5 Boxplot of median CT attenuation. The tissue can be differentiated by applying different X-ray spectra and analysing the differences in attenuation $\left(\mathrm{HU}_{80 \mathrm{kV}}-\mathrm{HU}_{140 \mathrm{kV}}\right)$ as demonstrated in Johnson et al. [7]. The boxplot shows the differentiation of ancient tissue and organic materials such as linen $(L)$, philtrum $(P)$, embalming material $(E M)$ and tongue $(T)$. The upper margin of the box represents the 75th percentile; the lower margin represents the 25 th percentile. The middle line of the box shows the median. The vertical lines with whiskers extending below and above the boxes indicate the minimal and maximal values. Open circles depict outliers



Fig. 6 Micro-CT of the neck, sample 1 from the Egyptian mummy head. The numbers 1 to 8 on the white line (length $7.3 \mathrm{~mm}$ ) depict higher density differences. The imaging dimension was $1,024 \times 1,024$, with a pixel size of $27 \mu \mathrm{m}$. The X-ray source voltage was $50 \mathrm{kV}$ at $503 \mu \mathrm{A}$

linen bandage and material with higher absorption, such as the embalming substance, are illustrated in sample 1 (white region under the linen). Soft tissue such as skin and fatty tissue could not be differentiated.

The greyscale intensity measurement of sample 1 is illustrated by the solid line in Fig 6. Three layers of linen bandage are indicated on the left side of Fig. 7 (three peaks in the diagram) in the distal direction of the neck sample. The thickness per linen layer was about $1.1 \mathrm{~mm}$. Consequently, the thickness of the whole wrapping of 12 layers was about $12 \mathrm{~mm}$. The greyscale intensity drop-off at positions 4, 5 and 7 (between the tissue layers) is caused by cracks in sample 1 . The peak between positions 4 and 5

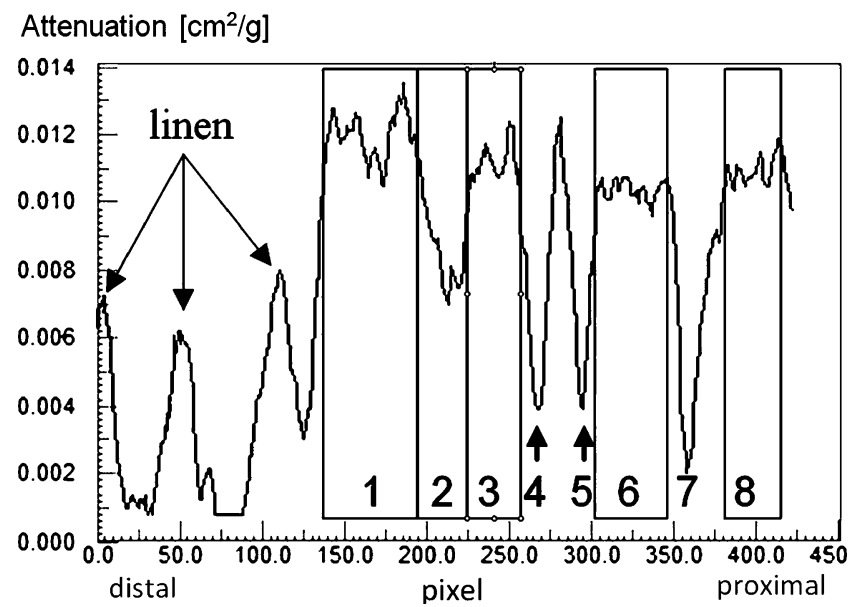

Fig. 7 Greyscale intensity line profile of sample 1 (numbers 1 to 8 ) showing the spatial compartments of the neck tissue (see also Fig. 6) 
demonstrates the higher X-ray absorption of remaining tissue.

The ring artefacts in Fig. 8 were caused by imperfect detector elements and could not be removed from the micro-CT image despite the correction algorithms implemented by the reconstruction tool. The embalming substances observed were visible as areas of white density on the micro-CT.

The presence of cracks and the embalming substance of ancient tissue is illustrated in Fig. 9. Moreover, sample 2 showed greater X-ray attenuation near the embalming material. The deep neck muscles under the trapezius could not be identified, e.g. the semispinalis capitis, the splenius capitis and the splenius cervicis.

The small FOV of micro-CT was a limitation in the macro-anatomical analysis. On the other hand, the tissue separation in group 3 was successfully carried out using the greyscale intensity line profile (Figs. 7, 9).

\section{Discussion}

\section{Dual-energy CT imaging}

Computed tomography imaging using a single energy has its limitation in the separation of smaller HU values (e.g. soft tissue).



Fig. 8 Micro-CT of neck sample 2 from the Egyptian mummy head. The linen layer below the sample marks the distal end of the neck. Note that the image was contrast-enhanced by histogram equalisation using ImageJ software (NIH, USA). The imaging dimension is $1,024 \times 1,024$, with a pixel size of $27 \mu \mathrm{m}$. The length of the solid line over the sample was about $11.3 \mathrm{~mm}$. The X-ray source voltage was $50 \mathrm{kV}$ at $503 \mu \mathrm{A}$

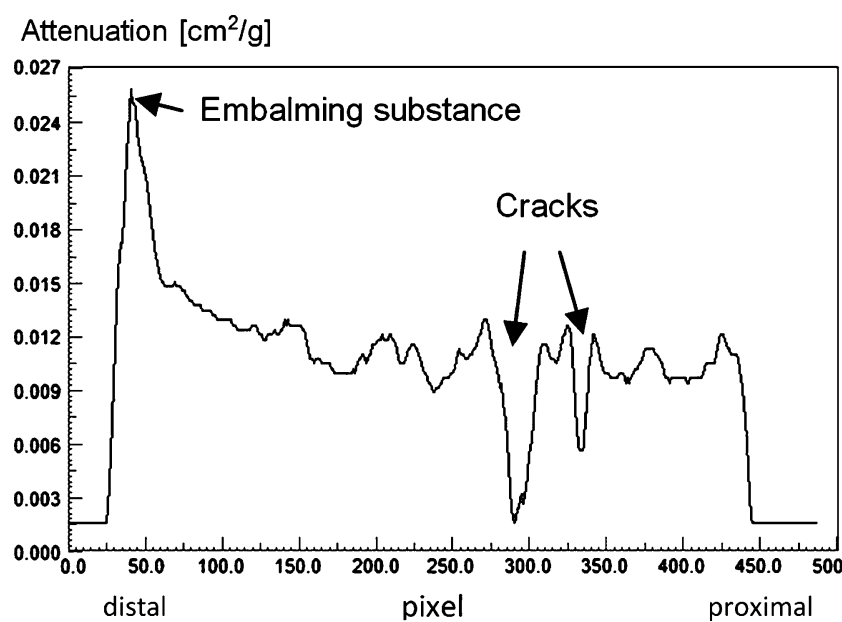

Fig. 9 Greyscale intensity line profile of sample 2, as depicted in Fig. 8

We found that HU-based DECT imaging with the use of two different energies has the potential to differentiate organic materials, such as linen, the philtrum, embalming material and the tongue.

The significant attenuation difference between the tissue groups $\{\mathrm{T}, \mathrm{EM}\}$ compared with their fully overlapped position in Fig. 4, revealed the value of DECT in the differentiation of organic materials with similar X-ray attenuation. On the other hand, Table 2 demonstrated that tissue separation (e.g. T, EM and $\mathrm{P}$ at $140 \mathrm{kVp}$ ) is barely possible using a single energy.

The non-significant difference in the mean intensity of the classes $\{\mathrm{T}, \mathrm{CB}\},\{\mathrm{T}, \mathrm{TB}\}$ and $\{\mathrm{EM}, \mathrm{TB}\}$ was influenced by measurements of heterogeneous bone tissue (Fig. 2). Smaller tissue layers such as deep neck muscles could not be differentiated due to insufficient spatial resolution of DECT as well as by the heterogeneity of dehydrated tissue and the fact that these tissues contain atoms with small atomic numbers, which at different energy levels show similar X-ray attenuation [11]. Two X-ray sources allow material differentiation relative to attenuation differences, which is especially effective for tissues with high atomic numbers, e.g. bone or calcified tissue.

Finally, the high temporal resolution of DECT as well as the lower radiation dose in comparison to conventional CT was not assessed in this study.

\section{Micro-CT imaging}

Micro-CT imaging with a field of view smaller than $20 \mathrm{~mm}$ is an invasive method in the archaeological context. A closer examination revealed that micro-CT with micrometer resolution has the potential to discriminate muscle tissue from inorganic material. However, greater spatial resolution and contrast is desirable to identify the content of individual 
tissue layers. Finally, micro-CT demonstrated the influence of the mummification process on the image quality of X-ray-based imaging, e.g. micro-cracks.

Quality aspects

In general, the dehydration process of tissue leads to more dense packing of collagen fibres and organelles in tissue [12].

Contrary to our findings, Johnson et al. [7] demonstrated with DECT imaging the successful differentiation of collagen in normal tissue. Nevertheless, we have to take into account the fact that ancient tissue is a shrunken, dry and brittle material with numerous air pockets and cracks inside the tissue. Therefore, the higher absorption differences in tissue as well as micro-cracks inside the material increase scattering during X-ray imaging. These physical alterations deteriorate the quality of the images significantly. On the other hand, DECT belongs to the fastest growing imaging technologies in this field, which further increases the future value of such imaging methods in paleoradiology.

This study was limited to a single mummy head. Further research is ongoing with imaging systems that overcome the aforementioned limitations through higher photon densities delivered from synchrotron sources.

\section{Conclusion}

Dual-energy CT imaging should be considered particularly for tissue discrimination with thick tissue layers such as bone, calcified tissue and embalming substance. The advantage of DECT over CT was observed in the study of mummified tissue. However, the improved temporal resolution and the improved diagnostic soft-tissue image quality of DECT was not a benefit in the context of ancient tissue discrimination [13]. Micro-CT with higher spatial resolution was able to identify microscopic anatomical changes in ancient tissue following the mummification process.
Acknowledgements We thank Franz Pfeiffer (Paul Scherrer Institute, Villigen, Switzerland) and Paul Stolzmann (Institute of Diagnostic Radiology, University Hospital Zurich, Switzerland) for performing the 3D imaging. The authors thank Jamie Harle (University of Liverpool, UK) for helpful comments on a preliminary draft of the paper.

\section{References}

1. Cockburn A, Cockburn E, Reyman TA. Mummies, disease and ancient cultures. Cambridge: Cambridge University Press; 1998.

2. Hoffman H, Torres WE, Ernst RD. Paleoradiology: advanced CT in the evaluation of nine Egyptian mummies. Radiographics. 2002;22:377-85.

3. Lewin PK, Harwood-Nash DC. X-ray computed axial tomography of an ancient Egyptian brain. IRCS Med Sci. 1977;5:78.

4. Aufderheide AC. Progress in soft tissue paleopathology. J Am Med Assoc. 2000;284(20):2571-3.

5. Stolzmann P. Dual-energy computed tomography for the differentiation of uric acid stones: ex vivo performance evaluation. Urol Res. 2008;36(3-4):133-8.

6. Graser A, Johnson TRC, Chandarana H, Macari M. Dual energy $\mathrm{CT}$ : preliminary observations and potential clinical applications in the abdomen. Eur Radiol. 2009;19:13-23.

7. Johnson TR, Krauß B, Sedlmair M, Grasruck M, Bruder H, Morhard D. Material differentiation by dual energy CT: initial experience. Eur Radiol. 2007;17(6):1510-7.

8. Rühli FJ, Kuhn G, Evison R, Müller R, Schultz M. Diagnostic value of micro-CT in comparison with histology in the qualitative assessment of historical human skull bone pathologies. Am J Phys Anthropol. 2007;133(4):1099-1111.

9. Motley G, Dalrymple N, Keesling C, Fischer J, Harmon W. Hounsfield unit density in the determination of urinary stone composition. Urology. 2001;58:170-3.

10. Gupta R, Markowitz Y, Berman L, Chapman P. High-resolution imaging of an ancient Egyptian mummified head: new insights into the mummification process. Am J Neuroradiol. 2008;29:705-13.

11. Persson A, Jakowski C, Engström E, Zachrisson H. Advances of dual source, dual-energy imaging in postmortem CT. Eur J Radiol. 2008;68(3):446-55.

12. Rylander CG, Stumpp OF, Milner TE, Kemp NJ, Mendenhall JM, Diller KR, et al. Dehydration mechanism of optical clearing in tissue. J Biomed Opt. 2006;11(4):041117.

13. Donnino R, Jacobs JE, Doshi JV, Hecht EM, Kim DC, Babb JS, et al. Dual-source versus single-source cardiac CT angiography: comparison of diagnostic image quality. Am J Roentgenol. 2009; 192:1051-6. 